\title{
Detection of mitochondrial DNA mutations by high-throughput sequencing in the blood of breast cancer patients
}

\author{
LIN HAI LI ${ }^{1,2^{*}}$, TAO KANG $^{3 *}$, LIDAN CHEN $^{2}$, WEIYUN ZHANG ${ }^{2}$, \\ YANG LIAO $^{2}$, JIANYUN CHEN ${ }^{2}$ and YULING SHI ${ }^{1,2}$ \\ ${ }^{1}$ Graduate School of Southern Medical University, Guangzhou, Guangdong 510515; ${ }^{2}$ Department of Laboratory Medicine, \\ Guangzhou General Hospital of Guangzhou Military Command, Guangzhou, Guangdong 510010; ${ }^{3}$ International Center \\ for Metabolic Diseases, Southern Medical University, Guangzhou, Guangdong 510515, P.R. China
}

Received June 27, 2013; Accepted September 20, 2013

DOI: $10.3892 /$ ijmm.2013.1559

\begin{abstract}
Mitochondrial DNA mutations have been identified in serveral types of cancer. In breast cancer, germline and somatic mitochondrial DNA (mtDNA) mutations have been identified. A number of mtDNA mutations in breast cancer have been identified in protein-coding regions (in protein-coding genes, such as ND2, COX3, ND4, ND5 and CytB). Mutations in these structure proteins cause impaired electron transport function and lead to electron leakage and increased reactive oxygen species (ROS) production, which in turn increases oxidative stress and oxidative damage to the mitochondria, as well as to cells. These data establish an association between mtDNA mutations and breast cancer; however, there is no reliable prediction of breast cancer predisposition or progression based on mtDNA mutation patterns thus far. In this study, we used high-throughput sequencing to detect mtDNA mutations in the blood of breast cancer patients. Some of these mutations may be used as potential markers for breast cancer diagnosis.
\end{abstract}

\section{Introduction}

Human mitochondrial DNA (mtDNA) is a $16.6 \mathrm{~kb}$ circular double-stranded DNA molecule, which is present at a high copy number per cell. Thirteen of the 85 subunits comprising the oxidative phosphorylation system are encoded by mtDNA, including subunits within complex I (ND1, ND2, ND3, ND4, ND4L, ND5, ND6), complex III (CytB), complex IV (COI, COII, COIII) and complex V (ATPase6, ATPase8). Human mtDNA also contains 24 genes encoding 22 transfer RNAs (tRNAs) and 2 ribosomal RNAs (rRNAs) which are essential

Correspondence to: Dr Yuling Shi, Graduate School of Southern Medical University, no. 1838 Guangzhoubei Road, Baiyun District, Guangzhou, Guangdong 510515, P.R. China

E-mail: yuling_shi@yahoo.com

${ }^{*}$ Contributed equally

Key words: mitochondrial DNA mutation, breast cancer, highthroughput sequencing, Ion Torrent for protein synthesis within the mitochondria. Another important region in mtDNA is termed the D-loop, which is the initial site of heavy chain replication and the promoters for heavy and light chain transcription $(1,2)$.

The mitochondrion functions as a powerhouse to generate adenosine triphosphate (ATP) through oxidative phosphorylation (OXPHOS). The mitochondrial OXPHOS also produces most of the cellular reactive oxygen species (ROS) at complexes I and III. It is believed that mtDNA mutations in the subunits of complexes I or III cause aberrant ROS production which may damage mtDNA, as well as nuclear DNA (3). In particular, the mitochondrion has relatively less sophisticated DNA protection and repair systems, and thus it is vulnerable to high mutation rates (4). The mitochondrion also plays central role in apoptosis $(5,6)$, cell proliferation (7) and calcium signaling (8).

Germline and somatic mtDNA mutations have been found in primary breast cancer. One mtDNA population polymorphism has been found to be associated with an increased risk of breast cancer, suggesting that germline mtDNA mutations may be important in the etiology of breast cancer. This mutation occurs in the ND3 gene at nt 10398 in which the 10398A allele was linked to an increased risk of invasive breast cancer in African-American women, compared with African-American women with the $10398 \mathrm{G}$ allele. The 10398 allele was an independent risk factor for breast cancer in African-American women but no association was detectable in Caucasian women. It was thought that this mutation results in increased oxidative stress (9). However, Setiawan et al argued that there was no association between the 10398 allele and breast cancer in African-American women; however, their study did not provide detail information on the materials and methods that were used (10). Parrella et al reported that mtDNA mutations were detected in $61 \%$ of patients using direct sequencing. The affected genes included ND4, ND5 and CytB (11). Tan et al reported that 14 of the 19 breast tumors (74\%) displayed at least one somatic mtDNA mutation. Twenty-seven somatic mutations were found and 22 of them occurred in the D-loop region. The affected genes included ND2, 16srRNA and ATPase6 (12). Gallardo et al reported mtDNA mutations of the COI gene in primary breast cancer. The mutation was expected to impair the interaction between 

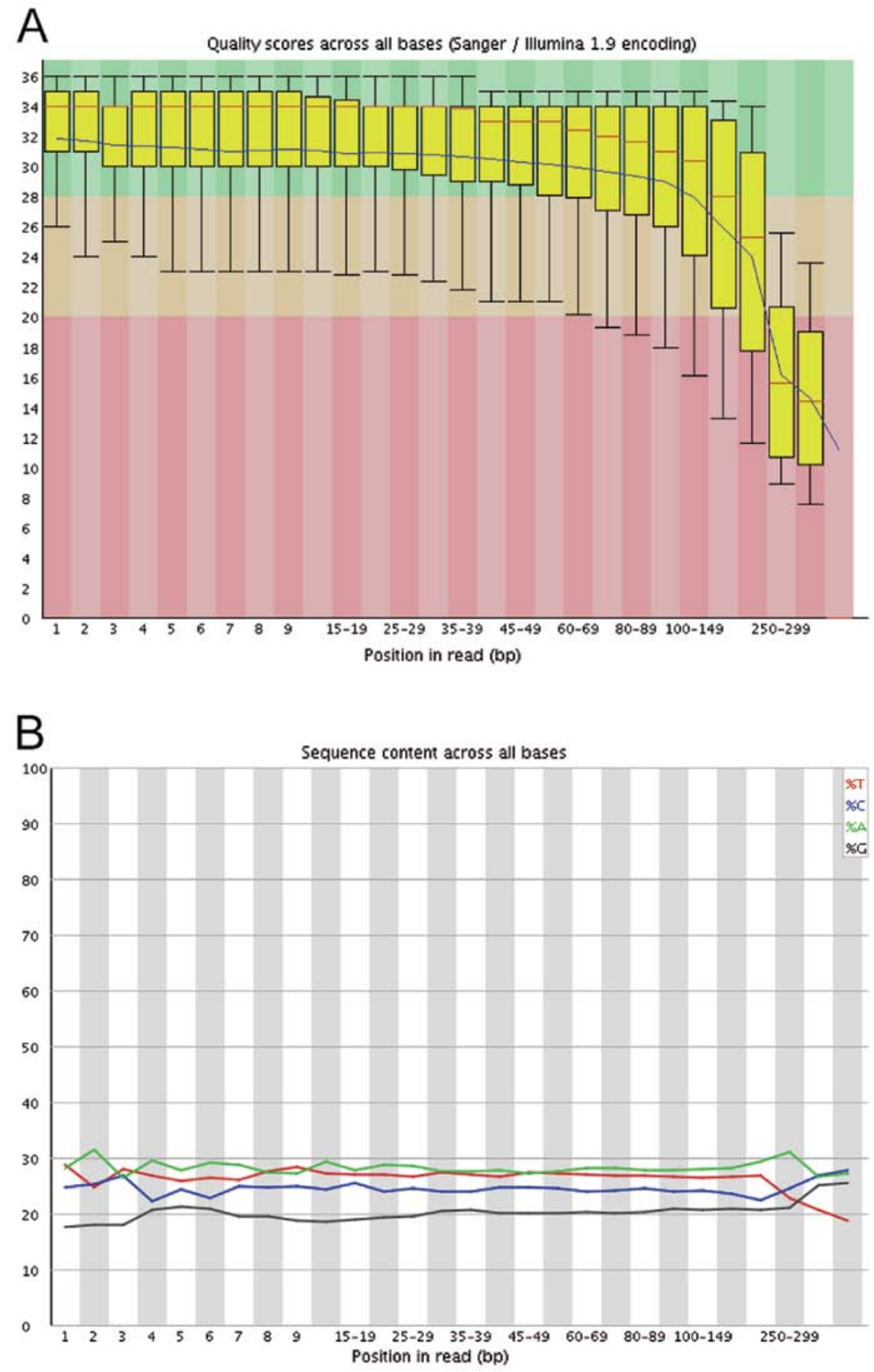

Figure 1. Quality control of sequencing data. (A) Quality scores across all bases. (B) Sequence content across all bases.

subunit I and II of cytochrome $c$ oxidase and the mutant had a reduced complex IV activity by $50 \%$ (13). Other studies have reported mtDNA mutations in the ND1, CoIII, tRNA-I and tRNA-T genes $(11,14)$. It is believed that mtDNA mutations in structure proteins of the mitochondria cause impaired electron transport function and lead to electron leakage and increased ROS production, which in turn increases oxidative stress and oxidative damage to the mitochondria in the process of transformation and cancer progression. There is no direct evidence on breast cancer to support the ROS theory thus far; however, there is experimental evidence on prostate cancer to support this theory. Petros et al reported that cybrids with pathogenic
mtDNA ATP6 T8993G generated tumors 7-fold larger than the wild-type (T8993T) cybrids. The mutant tumors generated significantly more ROS (15).

Studies in this field have established an association between mtDNA mutations and breast cancer. However, evidence for a direct association between these mtDNA mutations and breast cancer is still lacking in terms of the function of the mutant and the development of breast cancer. There has even been some debate between different research groups $(9,10,16)$. Moreover, there is no reliable prediction of breast cancer predisposition or progression based on mtDNA mutation patterns identified thus far. 
A

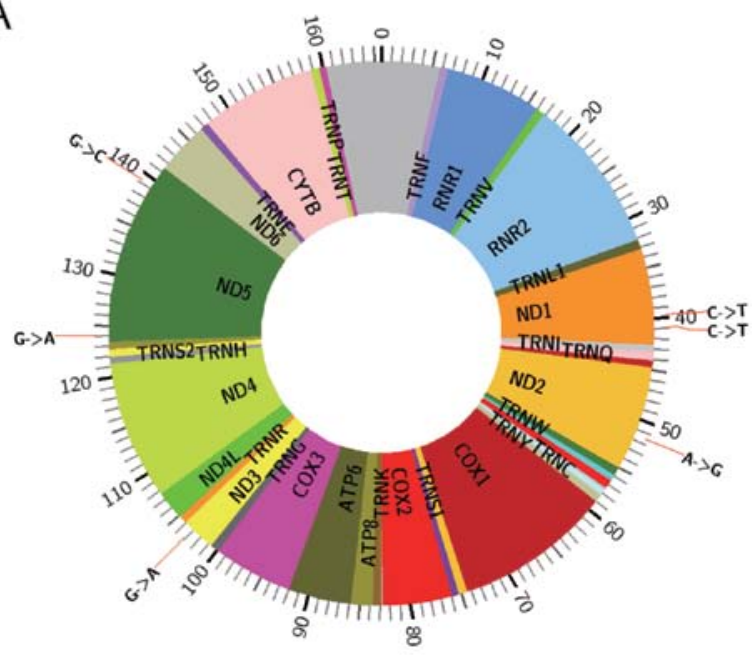

B

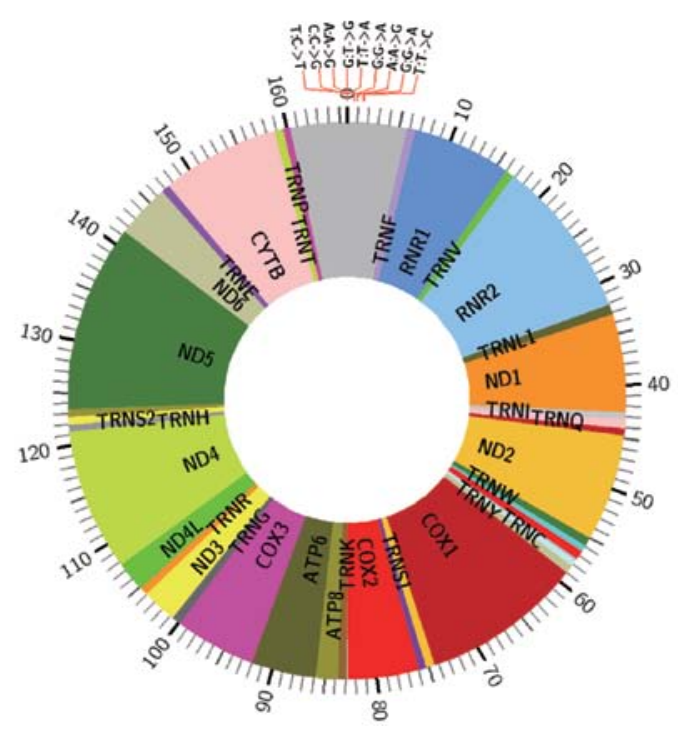

Figure 2. Single-nucleotide polymorphisms (SNPs) and heteroplasmy in pair 1 samples. (A) SNPs, (B) heteroplasmy.

\section{Materials and methods}

All blood samples were collected according to procedures approved by the Institutional Review Board of the Guangzhou General Hospital of Guangzhou Military Command. There were 58 blood samples from breast cancer patients and 58 samples from age-matched healthy individuals used in this study. mtDNA from total cellular DNA was enriched by PCR-based strategies.

In PCR-based enrichment, two sets of primers were designed to amplify amplicons that cover the mtDNA genome. The primers used in this study were as follos: Mito-8kb-A-Fwd, GACGGGCTCACATCACCCCATAA/Mito-8kb-A-Rev, GCG TACGGCCAGGGCTATTGGT and Mito-8kb-B-Fwd, GGT GGCTGGCACGAAATTGACC/Mito-8kb-B-Rev, GCC ACAACTAACCTCCTCGGACTCCT.

Purified, blunt-ended PCR products were subsequently fragmented by sonication. Fragmented DNA was then endrepaired, A-tailed and ligated with an adaptor oligonucleotide from the Ion Torrent genomic DNA library preparation kit
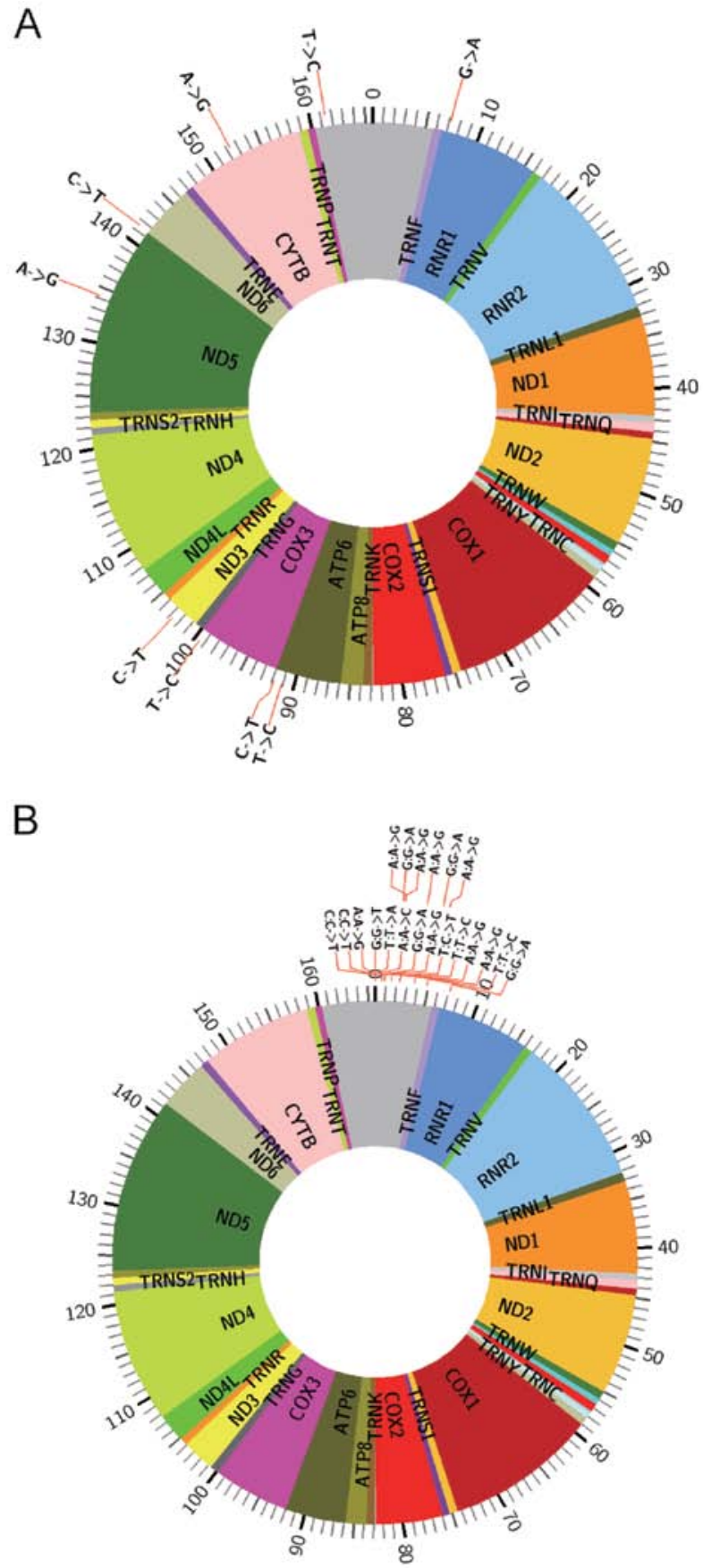

Figure 3. Single-nucleotide polymorphisms (SNPs) and heteroplasmy in pair 2 samples. (A) SNPs, (B) heteroplasmy.

(Life Technologies, South San Francisco, USA) in accordance with the manufacturer's instructions. Adaptor-ligated products were then size-selected by gel purification and sequenced by Ion Torrent PGM. The Mitomap (http://www.mitomap.org) and $\mathrm{mtDB}$ (http://www.genpat.uu.se/mtDB/) databases were used to identify sequence variants.

\section{Results}

High-throughput sequencing and quality control were performed. Quality scores were all above 200 and the sequence content was steady (Fig. 1). Thus, the sequence data were ready for use in further analysis. 

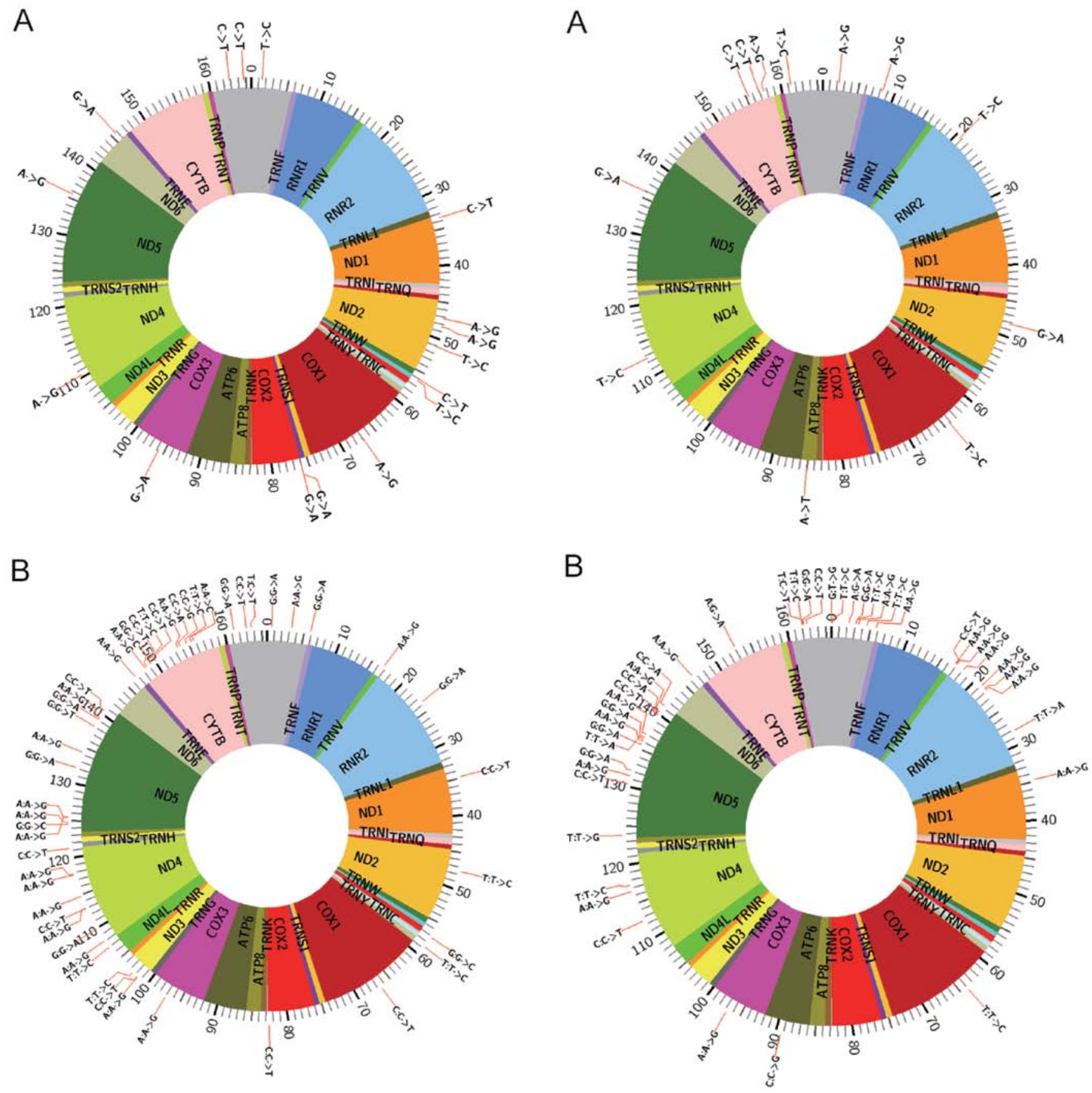

Figure 4. Single-nucleotide polymorphisms (SNPs) and heteroplasmy in pair 3 samples. (A) SNPs, (B) heteroplasmy.

In the following step, we compared data from the blood of breast cancer patients and the controls in 4 pairs of samples (Figs. 2-5). There were two pools of 28 blood samples from patients or controls in pair 1 and pair 2. For pair 3 and pair 4, there was one patient sample and one age-matched control. The reported variants were confirmed. In addition, we found a new mtDNA variant (13564 A>G). In this study, mitochondrial genomes were fully sequenced from blood DNA obtained from the patients with breast cancer and the normal controls. The new mtDNA variants may be potential biomarkers for the early detection of breast cancer. There are several advantages of using mtDNA as a potential biomarker for cancer-specific
Figure 5. Single-nucleotide polymorphisms (SNPs) and heteroplasmy in pair 4 samples. (A) SNPs, (B) heteroplasmy.

mutation studies. The genome is well characterized, with 16,568 bp harboring 37 genes. Secondly, a high copy number is an advantage over nuclear DNA for the detection of sequence variants. In addition, DNA repair is less efficient in the mitochondria than nuclear genomes; therefore, mutations are more easily identified.

\section{Discussion}

Evidence indicates an association between mtDNA mutations and breast cancer; however, there is no reliable prediction of breast cancer predisposition or progression based on mtDNA 
mutation patterns thus far. In this study, we compared mtDNA sequence data from the blood of breast cancer patients and controls. We confirmed the reported variants and found a new mtDNA variant (13564 A>G). As some studies have suggested, there may be two classes of cancer mtDNA mutations: tumorigenic mutations and adaptive mutations. Tumorigenic mutations would be advantageous in the initial phases of tumor growth, while the adaptive mutations would be advantageous in the late phases of tumor growth when the tumor becomes vascularized (17). Furthermore, since the two classes of mtDNA mutations have different functions in cancer cells, they may be expected to arise and be lost from the tumor cells at different phases during tumor growth. This could be the reason that the heteroplasmid $294 \mathrm{nt}$ ND1 deletion mtDNA has been shown to be present in $50 \%$ of the mtDNAs in primary renal cell carcinoma but absent in the subsequent metastatic tumors from the same patient $(17,18)$. The same may occur in breast cancer. Moreover, Rajasimha et al reported that the selection against pathogenic mtDNA mutations occurs in a stem cell population. They found that the percentage of pathogenic mtDNA mutations in the blood decreases exponentially over time compared with that in hematopoietic stem cells and leukocyte precursors (19). Therefore, it can be argued that primary breast cancer cells may not be the right cell population to detect tumorigenic mtDNA mutations. In other words, studies carried out on primary breast cancer cells to date have been unable to detect tumorigenic mtDNA mutations. Thus, perhaps it would be more effective to detect tumorigenic mtDNA mutations in breast cancer stem cells.

Solid evidence supports the hypothesis that breast cancer follows a cancer stem cell model. In 2003, Al-Hajj et al reported that they were able to identify and isolate a minority group of breast cancer cells using the surface marker, CD $44^{+} / \mathrm{CD} 24 \%$ low/Lin- Only a few of these cells (only 100 cells) were able to form tumors in mice, whereas of the rest cell population (tens of thousands cells) failed to form tumors. Furthermore, this tumorigenic subpopulation could be serially passaged and the subsequent tumors had a heterogeneity similar to the parental tumor: $\mathrm{CD}^{4} 4^{+} / \mathrm{CD} 24^{-} / \mathrm{low} / \mathrm{Lin}^{-}$tumorigenic cells, as well as the phenotypically diverse mixed populations of non-tumorigenic cells (20). There may be some concern in terms of species incompatibilities since they used a model in which human breast cancer cells were grown in immunocompromised mice. However, breast cancer stem cells were confirmed in following studies using mouse models of breast cancer. In 2008, Cho et al isolated and characterized cancer stem cells in MMTV-Wnt-1 murine breast tumors. They demonstrated that Thy $1^{+} \mathrm{CD} 24^{+}$ cancer cells were highly enriched cells capable of regenerating new tumors compared with the cells of the tumor that did not have this marker profile (21). Similarly, Zhang et al identified breast cancer stem cells in a p53-null mouse model of breast cancer using the $\mathrm{Lin}^{-} / \mathrm{CD} 29^{\text {high }} / \mathrm{CD} 24^{\text {high }}$ marker (22). Vaillant et al identified breast cancer stem cells in MMTV-Wnt-1, as well as in $\mathrm{p}^{53^{+/}}$mouse models using the luminal epithelial progenitor marker, CD61/ $\beta 3$ integrin (23). It seems that several sets of markers can be used to isolate breast stem cells, although the functions of these markers in stem cells remain unclear. In 2007, Ginestier et al isolated normal and malignant human mammary stem cells using aldehyde dehydrogenase (ALDH)1 as a marker. They demonstrated that normal and cancer human mammary epithelial cells with increased ALDH activity had stem cell/progenitor properties. In breast tumors, a high ALDH activity identified the tumorigenic cell fraction which was capable of self-renewal and generating tumors. The subsequent tumors recapitulated the heterogeneity of the parental tumor. Furthermore, the expression of ALDH1 detected by immunostaining correlated with a poor prognosis in a series of 577 breast carcinomas (24). In 2009, Charafe-Jauffret et al isolated breast cancer stem cells in 23 breast cancer cell lines using ALDH assay followed by FACS. They confirmed the stem cell properties of ALDH-positive populations in vitro and in NOD/SCID xenografts (25). Taken together, using highthroughput sequencing, the findings from our study provide new evidence to support the association between mtDNA mutations and breast cancer. As a next step, it would be helpful to detect tumorigenic mtDNA mutations in breast cancer stem cells to expand these findings.

\section{Acknowledgements}

This study was funded by grants from Guangdong Province, the China Science and Technology Development Project of Guangdong Province (2010B011300018-7) and the Natural Science Foundation of Guangdong Province (8451051501000491).

\section{References}

1. Attardi G and Schatz G: Biogenesis of mitochondria. Annu Rev Cell Biol 4: 289-333, 1988.

2. Lightowlers RN, Chinnery PF, Turnbull DM and Howell N: Mammalian mitochondrial genetics: heredity, heteroplasmy and disease. Trends Genet 13: 450-455, 1997.

3. Wallace DC: A mitochondrial paradigm of metabolic and degenerative diseases, aging, and cancer: a dawn for evolutionary medicine. Annu Rev Genet 39: 359-407, 2005.

4. DiMauro S and Schon EA: Mitochondrial DNA mutations in human disease. Am J Med Genet 106: 18-26, 2001.

5. Wang X: The expanding role of mitochondria in apoptosis. Genes Dev 15: 2922-2933, 2001.

6. Kroemer G and Reed JC: Mitochondrial control of cell death Nat Med 6: 513-519, 2000.

7. Rustin P: Mitochondria, from cell death to proliferation. Nat Genet 30: 352-353, 2002.

8. Babcock DF and Hille B: Mitochondrial oversight of cellular $\mathrm{Ca}^{2+}$ signaling. Curr Opin Neurobiol 8: 398-404, 1998.

9. Canter JA, Kallianpur AR, Parl FF and Millikan RC: Mitochondrial DNA G10398A polymorphism and invasive breast cancer in African-American women. Cancer Res 65: 8028-8033, 2005.

10. Setiawan VW, Chu LH, John EM, et al: Mitochondrial DNA G10398A variant is not associated with breast cancer in AfricanAmerican women. Cancer Genet Cytogenet 181: 16-19, 2008.

11. Parrella P, Xiao Y, Fliss M, et al: Detection of mitochondrial DNA mutations in primary breast cancer and fine-needle aspirates. Cancer Res 61: 7623-7626, 2001.

12. Tan DJ, Bai RK and Wong LJ: Comprehensive scanning of somatic mitochondrial DNA mutations in breast cancer. Cancer Res 62: 972-976, 2002.

13. Gallardo ME, Moreno-Loshuertos R, Lopez C, et al: $\mathrm{m} .6267 \mathrm{G}>\mathrm{A}$ : a recurrent mutation in the human mitochondrial DNA that reduces cytochrome $c$ oxidase activity and is associated with tumors. Hum Mutat 27: 575-582, 2006.

14. Zhu W, Qin W, Bradley P, Wessel A, Puckett CL and Sauter ER: Mitochondrial DNA mutations in breast cancer tissue and in matched nipple aspirate fluid. Carcinogenesis 26: 145-152, 2005.

15. Petros JA, Baumann AK, Ruiz-Pesini E, et al: mtDNA mutations increase tumorigenicity in prostate cancer. Proc Natl Acad Sci USA 102: 719-724, 2005.

16. Salas A, Yao YG, Macaulay V, Vega A, Carracedo A and Bandelt HJ: A critical reassessment of the role of mitochondria in tumorigenesis. PLoS Med 2: e296, 2005. 
17. Brandon M, Baldi P and Wallace DC: Mitochondrial mutations in cancer. Oncogene 25: 4647-4662, 2006.

18. Horton TM, Petros JA, Heddi A, et al: Novel mitochondrial DNA deletion found in a renal cell carcinoma. Genes Chromosomes Cancer 15: 95-101, 1996.

19. Rajasimha HK, Chinnery PF and Samuels DC: Selection against pathogenic mtDNA mutations in a stem cell population leads to the loss of the 3243A-->G mutation in blood. Am J Hum Genet 82: 333-343, 2008.

20. Al-Hajj M, Wicha MS, Benito-Hernandez A, Morrison SJ and Clarke MF: Prospective identification of tumorigenic breast cancer cells. Proc Natl Acad Sci USA 100: 3983-3988, 2003.

21. Cho RW, Wang X, Diehn M, et al: Isolation and molecular characterization of cancer stem cells in MMTV-Wnt-1 murine breast tumors. Stem Cells 26: 364-371, 2008.
22. Zhang M, Behbod F, Atkinson RL, et al: Identification of tumorinitiating cells in a p53-null mouse model of breast cancer. Cancer Res 68: 4674-4682, 2008

23. Vaillant F, Asselin-Labat ML, Shackleton M, Forrest NC, Lindeman GJ and Visvader JE: The mammary progenitor marker CD61/beta3 integrin identifies cancer stem cells in mouse models of mammary tumorigenesis. Cancer Res 68: 7711-7717, 2008.

24. Ginestier C, Hur MH, Charafe-Jauffret E, et al: ALDH1 is a marker of normal and malignant human mammary stem cells and a predictor of poor clinical outcome. Cell Stem Cell 1: 555-567, 2007.

25. Charafe-Jauffret E, Ginestier C, Iovino F, et al: Breast cancer cell lines contain functional cancer stem cells with metastatic capacity and a distinct molecular signature. Cancer Res 69: 1302-1313, 2009 